\title{
Intrasubband and Intersubband Electron Relaxation in Semiconductor Quantum Wire Structures
}

\author{
Marcos R.S. Tavares and S. Das Sarma \\ Department of Physics, University of Maryland, College Park, MD 20742-4111, \\ $U S A$ \\ Guo-Qiang Hai \\ Instituto de Física de São Carlos, Universidade de São \\ Paulo, São Carlos, SP 13560-970, Brazil
}

\begin{abstract}
We calculate the intersubband and intrasubband many-body inelastic Coulomb scattering rates due to electron-electron interaction in two-subband semiconductor quantum wire structures. We analyze our relaxation rates in terms of contributions from inter- and intrasubband charge-density excitations separately. We show that the intersubband (intrasubband) charge-density excitations are primarily responsible for intersubband (intrasubband) inelastic scattering. We identify the contributions to the inelastic scattering rate coming from the emission of the single-particle and the collective excitations individually. We obtain the lifetime of hot electrons injected in each subband as a function of the total charge density in the wire.
\end{abstract}

Typeset using REVTEX 


\section{INTRODUCTION}

Semiconductor quantum wire structures, based mostly on GaAs-AlGaAs systems, have been studied intensively for the last ten years as systems of potential technological interest (e.g. quantum wire lasers), and also because of their fundamental significance as examples of quasi-one-dimensional (Q1D) electron liquids. Among the important research milestones in semiconductor quantum wires are the observation [1] of one dimensional plasmons via the inelastic light scattering spectroscopy and the verification of the predicted acoustic linear plasma dispersion relation [2] in one dimension, the observation of pronounced one dimensional Fermi edge singularities in the optical spectra [3], the quantum wire excitonic laser operation [4] and its theoretical understanding [5]. With improving materials growth and nano-fabrication techniques one expects a wide range of one dimensional experimental phenomena and projected applications in semiconductor quantum wire systems. Many of the projected applications such as ballistic electron transistors, quantum wire-based infrared photo-detectors and lasers, quantum wire THZ oscillators and modulators, will utilize fast carriers (injected or excited) in doped quantum wires as the active device element. Effective control and manipulation of these fast electrons in doped quantum wire systems are therefore essential in the projected quantum wire opto-electronic applications. One of the most crucial physical processes that will limit the quantum wire opto-electronic applications is the relaxation of these fast electrons. The main ultrafast mechanism controlling the relaxation process is the electron-electron interaction, which is also a many-body process of fundamental importance in electronic systems. In this paper, we develop a many-body theory for the electron-electron interaction induced ultrafast relaxation in semiconductor quantum wires with more than one quantized subband occupied. We consider only the ultrafast electronelectron interaction induced relaxation in this article, neglecting the weaker electron-phonon Fröhlich coupling. The electron-phonon coupling may be considered to be approximately included in our theory by taking the effective mass entering the theory to be the polaronic band mass (including the electron-LO phonon interaction) and the background dielectric 
constant to be the static low frequency lattice dielectric constant (rather than the dynamic high frequency dielectric constant). We restrict ourselves to Coulomb scattering because the fastest relaxation time scales are controlled by the inter-electron Coulomb interaction.

We mention that semiconductor quantum wires in the strict one dimensional limit with only one occupied subband are extremely difficult to fabricate. Thus the typical experimental quantum wires would have a few occupied subbands and scattering between these subbands effectively destroys their strict one dimensionality. The work presented in this article takes a first step toward developing a full many-body theory for quantum wires with many occupied subbands by considering carefully the situation with two occupied subbands and by analyzing the resultant relaxation rates in terms of intrasubband (one dimensional) and intersubband (non-one dimensional) scattering contributions. In addition, we calculate single-particle and collective mode contributions to the relaxation rates separately. Our calculations can be directly compared with experimentally measured linewidths (e.g. the spectral width in tunneling measurements [6] or in the femtosecond spectroscopy [7] ) or band broadenings and with various relaxation rates entering device modelling considerations.

Intra- and intersubband relaxation of electrons in Q1D doped semiconductor quantum wires are determined by their inelastic lifetime which is inversely proportional to the inelastic Coulomb scattering rate. Due to the Coulomb interaction, electrons in the quantum wires may be scattered and, as a result, collective ('plasmons') and single-particle excitations are emitted. Such lifetime calculations have earlier been carried out in 2D electrons systems [8,9], and have been interpreted in terms of plasmon emission processes. In contrast to $2 \mathrm{D}$ electrons gases, a gap shows up in the intersubband single-particle excitation continuum in Q1D quantum wires with two occupied subbands. [2,10 12] Furthermore, an extra intersubband plasmon mode appears within such a gap. It was also shown previously that, for a two-subband quantum wire, [13] the intersubband inelastic-scattering rates due to plasmon modes and single-particle excitations do not exist if the intersubband coupling is neglected. But the intrasubband inelastic-scattering rates were found consist of three contributions: that coming from the emission of plasmon modes in the (i) first and (ii) 
second subband; and the emission of a (iii) single-particle excitation in the second subband. So far in the literature the intersubband coupling in quantum wires has been considered irrelevant for electron relaxation in the conduction band. However, as the second subband becomes occupied, electrons in different subbands may interact strongly with each other, and as a consequence, intersubband coupling should in general be taken into account. In this paper we calculate the intra- and intersubband inelastic-scattering rates of electrons in a two-subband quantum wires with a small energy separation between the two subbands. We neglect the higher lying subbands to reduce computational complications. A generalization of our theory to many subbands is, in principle, possible. Currently there are no direct experimental observations of intersubband lifetimes in multisubband quantum wires, but our calculations should be relevant to a large number of projected applications.

We develop our theory for the inelastic Coulomb scattering treating the dynamical screening of the Q1D electron system within the framework of the random-phase approximation (RPA). The RPA has been shown to be an excellent approximation in studying chargedensity excitations in Q1D doped semiconductors by virtue of the approximate vanishing of all vertex corrections to the one-dimensional irreducible polarizability. [2, 14] In addition to the 1D intrasubband plasmons, the intersubband collective and single-particle excitations in the Q1D system also provide relaxation channels through which the hot electrons in the conduction band relax. We show that the inelastic-scattering rate from the second to the first subband can only occur through the emission of an intersubband plasmon with single-particle excitations not participating at all in this intersubband relaxation process, whereas emission of both collective and single-particle excitations contributes to the inelastic scattering from the first to the second subband.

This paper is organized as follows. In Sec. II we describe our theoretical approach. In Sec. III we present our numerical results for the inelastic scattering rates in a two-subband quantum wire. We conclude with a summary in Sec. IV. 


\section{MODEL AND EQUATIONS}

The single electronic states in our theory are calculated by considering a two-dimensional system in the $x y$ plane subjected to an additional confinement in the $y$-direction creating a GaAs/AlGaAs quantum wire in the $x$-direction. The confinement potential in the $y$ direction is taken to be of a finite square well type of barrier height $V_{0}$ and well width $W$. We assume the confinement potential creating the 2D confinement to be sufficiently strong compared with the 1D confinement potential and assume the 2D system to be ideal, i.e. of zero thickness in the third (z) direction. The 1D subband energies $E_{n}$ and the wave functions $\phi_{n}(y)$ are obtained from the numerical solution of the one-dimensional Schrödinger equation in the $y$-direction (the value of the electron effective mass throughout this paper is $m^{*}=$ $\left.0.07 m_{e}\right)$. We restrict ourselves to the case where $n=1,2$ and define $\omega_{0}=E_{2}-E_{1}$ as being the intersubband energy gap between the two subbands. For a symmetric confinement potential, the two lowest wave functions $\phi_{1}(y)$ and $\phi_{2}(y)$ are the usual symmetric and antisymmetric levels, respectively. We consider throughout this paper the confinement potential being of a barrier height $V_{0}=100 \mathrm{meV}$ and well width $W=500 \AA$, which leads to $\omega_{0} \simeq 5.37 \mathrm{meV}$. Then, the second subband becomes populated at $1 \mathrm{D}$ electron density $N_{e}=6.3 \times 10^{5} \mathrm{~cm}^{-1}$. As mentioned in the introduction, we neglect inelastic scattering due to emission of phonons. Such a procedure is reasonable since the emission of an LO phonon, for example in GaAs, requires the electron energy to be at least $\hbar \omega_{L O} \simeq 36 \mathrm{meV}$ which is much larger than the characteristic inter-subband energy $\omega_{0}$ of our quantum wire. We will restrict ourselves to situations where phonon emission processes are not important. We take $\hbar=1$ throughout this paper unless stated otherwise.

As we mentioned in the introduction, intra- and intersubband relaxation of fast electrons in two-subband quantum wire structures can be studied in determining their inelastic Coulomb scattering rate $\sigma_{n n^{\prime}}(k)$, where $n, n^{\prime}=1,2$. Due to Coulomb interaction, these electrons, initially in a subband $n$ with momentum $k$, can be scattered to a subband $n^{\prime}$ with momentum $k^{\prime}$ through emission of both plasmons and single-particle excitations. Within 
the so-called GW approximation, Vinter [15] originally showed that, at zero temperature, the inelastic Coulomb scattering rate $\sigma_{n n^{\prime}}(k)$ of electrons in multisubband structures can be obtained from the imaginary part of the retarded electron self-energy neglecting higher order vertex corrections. This approximation is extensively employed in calculating electronic many-body effects and, in particular, has been used in studying injected electron lifetimes in semiconductor quantum wire structures in the strict one-dimensional limit (or equivalently in determining the intrasubband inelastic scattering rate of electrons in the first quantized subband), 13,16,17 as well as in coupled parallel quantum wires. 18 Within the GW approximation, the multisubband inelastic scattering rate $\sigma_{n n^{\prime}}(k)$ of fast electrons in quasi-one-dimensional quantum wires at zero temperature is given by

$$
\begin{aligned}
& \sigma_{n n^{\prime}}(k)=\frac{1}{2 \pi} \int d q \operatorname{Im}\left\{V_{n n^{\prime} n^{\prime} n}^{s}\left[q, \xi_{n^{\prime}}(k+q)-\xi_{n}(k)\right]\right\} \\
& \times\left\{\theta\left(\xi_{n}(k)-\xi_{n^{\prime}}(k+q)\right)-\theta\left(-\xi_{n^{\prime}}(k+q)\right)\right\},
\end{aligned}
$$

where $\theta(x)$ is the standard step function, $V_{n n^{\prime} n^{\prime} n}^{s}(q, \omega)$ the dynamically screened electronelectron Coulomb potential with $q$ being the $1 \mathrm{D}$ wavevector and $\omega$ the mode frequency, and $\xi_{n}(k)=\hbar^{2} k^{2} / 2 m^{*}+E_{n}-E_{F}$ the electron energy with respect to the Fermi energy $E_{F}$. The screened Coulomb potential in Eq. (11) is related to the multisubband dielectric function $\varepsilon_{n n^{\prime} m m^{\prime}}(q, \omega)$ and the bare electron-electron interaction potential $V_{n n^{\prime} m m^{\prime}}(q)$ through the generalized RPA equation [19]

$$
\sum_{l l^{\prime}=1,2} \epsilon_{l l^{\prime} n n^{\prime}}(q, \omega) V_{l l^{\prime} m m^{\prime}}^{s}(q, \omega)=V_{n n^{\prime} m m^{\prime}}(q)
$$

with $m, m^{\prime}=1,2$. The bare electron-electron potential $V_{n n^{\prime} m m^{\prime}}(q)$, which is the 2-particle matrix element of $1 \mathrm{D}$ Coulomb interaction in the $\phi_{n}(y)$ basis, is calculated by using the numerical solution of the electron wavefunction $\phi_{n}(y)$. The dielectric function

$$
\varepsilon_{n n^{\prime} m m^{\prime}}(q, \omega)=\delta_{n m} \delta_{n^{\prime} m^{\prime}}-\Pi_{n n^{\prime}}(q, \omega) V_{n n^{\prime} m m^{\prime}}(q)
$$

is calculated within the RPA, where 


$$
\Pi_{n n^{\prime}}(q, \omega)=\frac{1}{\pi} \int d k \frac{n_{F}\left[\xi_{n}(k)\right]-n_{F}\left[\xi_{n^{\prime}}(k+q)\right]}{\xi_{n}(k)-\xi_{n^{\prime}}(k+q)+\omega}
$$

is the noninteracting irreducible polarizability function. Here, $n_{F}(E)$ is the Fermi distribution function. The polarizability $\Pi_{n n^{\prime}}(q, \omega)$ characterizes the bare electron-hole bubble polarization diagram and is written for the system free from any impurity scattering. The impurity scattering effects can be introduced diagrammatically by including impurity ladder diagrams in the electron Green's function. These diagrams are responsible for level broadening, or equivalently, for a phenomenological damping constant $\gamma=e^{2} / 2 m^{*} \mu$ mainly induced by scattering of electrons due to impurity centers, with $\mu$ being the carrier mobility in the sample. The exact expression for the polarizability within this diagrammatic approach can be obtained by using a particle-conserving approximation for arbitrary values of $q$ and $\omega$, given by Mermin. [20] In the limit $\gamma \rightarrow 0$, the Mermin's polarizability is found to be identical to Eq. (田) with the frequency $\omega^{2} \rightarrow \omega(\omega+i \gamma)$. In this paper, we take the impurity scattering induced broadening $\gamma$ as being a very small phenomenological damping parameter which allows us working in the limit $\gamma \rightarrow 0$. We are therefore restricting ourselves to high mobility quantum wires with small level broadening.

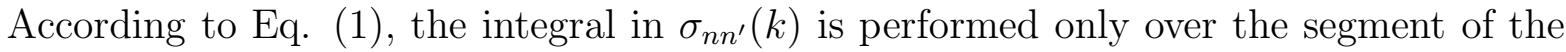
curve

$$
\omega_{k}^{n n^{\prime}}(q)=\xi_{n^{\prime}}(k+q)-\xi_{n}(k)
$$

which lies inside those regions where

$$
\theta\left[-\omega_{k}^{n n^{\prime}}(q)\right]-\theta\left[-\xi_{n^{\prime}}(k+q)\right] \neq 0 .
$$

We need to consider, therefore, just that segment of $\omega_{k}^{n n^{\prime}}(q)$ which lies in the region where the condition defined in Eq. (6) is satisfied. The inelastic scattering rates vanish outside these regions which means that the momentum and energy conservation cannot be simultaneously obeyed for those values of $\left(k, n, n^{\prime}, k+q\right)$. The inelastic scattering rate $\sigma_{n n^{\prime}}(k)$ is a nonvanishing term if the segment $\omega_{k}^{n n^{\prime}}(q)$ either lies in the continuum representing single-particle 
excitations or intercepts the lines representing collective excitations (plasmons) in the $q-\omega$ plane.

\section{NUMERICAL RESULTS AND DISCUSSIONS}

\section{A. Collective and single-particle excitation modes}

In our quantum wires we consider a symmetric square well potential characterizing the

$y$-direction confinement. Due to this symmetry, the two lowest wave functions $\phi_{1}(y)$ and $\phi_{2}(y)$ are symmetric and antisymmetric functions of $y$, respectively. As a result, the bare electron-electron Coulomb potential $V_{n n^{\prime} m m^{\prime}}(q)$ vanishes if $n+n^{\prime}+m+m^{\prime}$ is an odd number. Moreover

$$
V_{n n^{\prime} m m^{\prime}}(q)=V_{n^{\prime} n m m^{\prime}}(q)=V_{n n^{\prime} m^{\prime} m}(q)=V_{n^{\prime} n m^{\prime} m}(q)
$$

On the other hand, the dispersion of the collective plasmon modes is given by the zeros of the determinant of the dielectric tensor defined by Eq. (3), i.e., det $\left|\varepsilon_{n n^{\prime} m m^{\prime}}(q, \omega)\right|=0$. By using the symmetry properties of $V_{n n^{\prime} m m^{\prime}}(q)$ into this determinant, one can show that the intersubband plasmon modes are given by the roots of

$$
\varepsilon_{\text {inter }}=1-V_{1212}\left[\Pi_{12}+\Pi_{21}\right]=0
$$

whereas the intrasubband plasmon modes are obtained by the roots of

$$
\varepsilon_{\text {intra }}=\left[1-V_{1111} \Pi_{11}\right]\left[1-V_{2222} \Pi_{22}\right]-V_{1122}^{2} \Pi_{11} \Pi_{22}=0 .
$$

It is apparent that the intrasubband plasmon modes do not couple with the intersubband ones. This is, of course, a direct result of our symmetric confinement model which remains a reasonable model even in the presence of small asymmetries in the 1D confinement.

First, we consider a high electron density $\left(N_{e}=N_{1}=10^{6} \mathrm{~cm}^{-1}\right)$ in which case both the subbands are populated. The Fermi wavevectors in the first and second subbands are $k_{F 1}=1.09 \times 10^{6} \mathrm{~cm}^{-1}$ and $k_{F 2}=0.47 \times 10^{6} \mathrm{~cm}^{-1}$, respectively. In this case, two intrasubband 
and two intersubband plasmon modes exist corresponding to the two subbands. Fig. 1(a) shows the dispersion relations of the two intersubband plasmon modes $(1,2)$ and $(1,2)^{\prime}$ obtained from Eq. (8). The shadow areas indicate the intersubband single-particle excitation $\left(\mathrm{SPE}_{12}\right)$ continua where $\operatorname{Im}\left\{\Pi_{12}(q, \omega)\right\} \neq 0$. The intersubband $\mathrm{SPE}_{12}$ is of a finite frequency $\left(\omega=\omega_{0}\right)$ at $q=0$. The occupation of the second subband opens up a gap in the $\mathrm{SPE}_{12}$ continuum where the low-frequency intersubband plasmon mode $(1,2)^{\prime}$ appears. We also see a large depolarization shift of the high-frequency intersubband plasmon mode $(1,2)$. In Fig. 1(b) we show the dispersion relation of the intrasubband plasmon modes $(1,1)$ and $(2,2)$ obtained from the Eq. (9)). The intrasubband single-particle excitation $\mathrm{SPE}_{11}\left(\mathrm{SPE}_{22}\right)$ continuum where, $\operatorname{Im}\left\{\Pi_{11}(q, \omega)\right\} \neq 0\left(\operatorname{Im}\left\{\Pi_{22}(q, \omega)\right\} \neq 0\right)$, is also presented in the figure. The undamped second subband intrasubband plasmon mode $(2,2)$, lying in the gap between the $\mathrm{SPE}_{11}$ and $\mathrm{SPE}_{22}$ continua, has a linear energy dispersion as $q \rightarrow 0$. When this plasmon mode enters the $\mathrm{SPE}_{11}$ continuum, it is Landau damped because it can decay by emitting $\mathrm{SPE}_{11}$ excitations in the lowest subband. The plasmon mode $(1,1)$ representing the collective charge-density excitation in the first subband has an energy proportional to $q|\ln (q W)|^{1 / 2}$. Notice that, due to the symmetry of the system, the intersubband single-particle excitations do not damp the intrasubband plasmon modes and vice versa. In Fig. 2 we show the plasmon dispersion relations for a lower total electron density $N_{e}=N_{2}=0.40 \times 10^{6} \mathrm{~cm}^{-1}$. In this case, only the first subband is occupied. The corresponding 1D Fermi wavevector is $k_{F 1}=0.63 \times 10^{6} \mathrm{~cm}^{-1}$. Obviously, the plasmon mode $(2,2)$ as well as the continuum $\mathrm{SPE}_{22}$ do not exist since the second subband is empty. By analyzing the collective and single-particle excitation spectra in Figs. 1 and 2 and comparing them with $\omega_{k}^{n n^{\prime}}(q)$ defined in Eq. (5) we are able to figure out the contributions of different scattering mechanisms to the total inelastic-scattering rate. 


\section{B. Intrasubband scattering rate}

According to Eq. (1), the intrasubband inelastic scattering rates $\sigma_{11}(k)$ and $\sigma_{22}(k)$ are defined in terms of the imaginary part of the dynamically screened Coulomb potentials

$$
V_{1111}^{s}=\frac{V_{1111}\left(1+V_{2222} \Pi_{22}\right)-V_{1122}^{2} \Pi_{22}}{\varepsilon_{\text {intra }}}
$$

and

$$
V_{2222}^{s}=\frac{V_{2222}\left(1+V_{1111} \Pi_{11}\right)-V_{1122}^{2} \Pi_{11}}{\varepsilon_{\text {intra }}},
$$

respectively. These expressions are obtained from Eq. (2) and demonstrate that the contributions to $\sigma_{11}(k)$ and $\sigma_{22}(k)$ come from three sources. The first one is the emission of the intrasubband single-particle excitations. We numerically evaluate the integral in Eq. (11) only in those regions where $\operatorname{Im} \Pi_{22} \neq 0\left(\operatorname{Im} \Pi_{11} \neq 0\right)$. In Fig. 3(a) the thick-solid (thickdashed) line shows the intrasubband inelastic-scattering rate $\sigma_{11}(k)\left(\sigma_{22}(k)\right)$ due only to the emission of the single-particle excitations in the $\mathrm{SPE}_{22}\left(\mathrm{SPE}_{11}\right)$ continuum. We take the impurity broadening or the phenomenological damping constant $\gamma=10^{-3}$ meV corresponding to samples with very high electron mobility. All other parameters are the same as in the beginning of Sec. II. Our results show that the intrasubband inelastic scattering in one subband takes place through the emission of a single-particle excitation in the other subband. In fact, we verified that $\omega_{k}^{n n}(q)$ defined in Eq. (5) never crosses the $\mathrm{SPE}_{n n}$ continuum in the $q-\omega$ plane for $n=1$ and 2 independent of the value of $k$. This is the reason why the $\mathrm{SPE}_{11}$ and $\mathrm{SPE}_{22}$ continua do not contribute to $\sigma_{11}(k)$ and $\sigma_{22}(k)$, respectively. We also verified that the curve $\omega_{k}^{11}(q)$ is completely out of the $\mathrm{SPE}_{22}$ continuum for $k \geq k_{F 2}$ and, consequently, the contribution of the $\mathrm{SPE}_{22}$ to $\sigma_{11}(k)$ vanishes. On the other hand, the contribution of the $\mathrm{SPE}_{11}$ to $\sigma_{22}(k)$ starts at $k=k_{F 1}$ where the curve $\omega_{k_{F 1}}^{22}(q)$ lies exactly on the lower edge of the $\mathrm{SPE}_{11}$ continuum, entering the continuum for momentum $k>k_{F 1}$. Thus, the $\mathrm{SPE}_{22}$ contributes to $\sigma_{11}(k)$ from $k=0$ up to $k=k_{F 2}$, while the onset of the scattering

$\sigma_{22}(k)$ via emission of a single-particle excitation inside the continuum $\mathrm{SPE}_{11}$ occurs at the 
threshold $k=k_{F 1}$. The thin-dashed line in Fig. 3(a) indicates the $\mathrm{SPE}_{11}$ contribution to the scattering rate $\sigma_{22}(k)$ for an electron in a quantum wire of a density $N_{e}=N_{2}$. At this density the second subband is empty and as a consequence the $\operatorname{SPE}_{22}$ contribution to $\sigma_{11}(k)$ does not exist.

According to Eqs. (10) and (11), the other two sources contributing to $\sigma_{11}(k)$ and $\sigma_{22}(k)$ are the two zeros of $\varepsilon_{\text {intra }}$, i.e. the emission of intrasubband plasmon modes $(1,1)$ and $(2,2)$. In Fig. 3(b) we show the intrasubband inelastic-scattering rate $\sigma_{n n}(k)$ due only to the intrasubband plasmon modes $(n, n)$ with $n=1$ and 2 . These results are obtained by excluding the single particle excitation regions in the $q-\omega$ plane where $\operatorname{Im}\left[\Pi_{22}\right] \neq 0$ and $\operatorname{Im}\left[\Pi_{11}\right] \neq 0$ from the numerical integration characterizing $\sigma_{11}(k)$ and $\sigma_{22}(k)$, respectively. For $N_{e}=N_{1}$, the onset of both intrasubband scattering via emission of the plasmon mode $(1,1)$ occurs at the threshold $k=k_{c}^{11} \simeq 2.07 \times 10^{6} \mathrm{~cm}^{-1}$ corresponding to an interception of the curve $\omega_{k}^{11}(q)$, as well as $\omega_{k}^{22}(q)$, with the mode $(1,1)$ at $q=q_{c}^{11} \simeq 0.55 \times 10^{6} \mathrm{~cm}^{-1}$. Indeed, the emission of the plasmon mode $(1,1)$ is the most important contribution to the intrasubband scattering rates due to its significant spectral weight at $q=q_{c}^{11}$ leading to a huge divergence at $k=k_{c}^{11}$. The onset of scattering due to the plasmon mode $(2,2)$ occurs at the threshold $k=k_{F 2}\left(k_{c}^{22} \simeq 0.8 \times 10^{6} \mathrm{~cm}^{-1}\right)$ for an electron in the first (second) subband. Furthermore, the inelastic-scattering rate $\sigma_{22}(k)$ at $k=k_{F 1}$ is non-zero since $\omega_{k_{F 1}}^{22}(q)$ lies just on the lower edge of the $\mathrm{SPE}_{11}$ continuum where the plasmon $(2,2)$ contributes to scattering. But it no longer contributes to $\sigma_{22}(k)$ for $k>k_{F 1}$ due to the Landau damping. The fact that $\sigma_{22}(k)$ at $k=k_{F 1}$ is finite means that a plasmon mode $(2,2)$ may be emitted when the electron has an energy $\xi_{2}\left(k_{F 1}\right)$. Finally, the thin-solid (thin-dashed) line shows the contribution of the plasmon mode $(1,1)$ to $\sigma_{11}(k)\left(\sigma_{22}(k)\right)$ for $N_{e}=N_{2}$ where the second subband is empty. As expected, the contribution coming from the plasmon mode $(2,2)$ is absent in this case. 


\section{Intersubband scattering rates}

The definition in Eq. (1) tells us that the intersubband inelastic scattering rates $\sigma_{12}(k)$ and $\sigma_{21}(k)$ are obtained in terms of the imaginary part of the screened Coulomb potential

$$
V_{1221}^{s}=\frac{V_{1212}}{\varepsilon_{\text {inter }}}
$$

Notice that, according to Eq. (2), $V_{2112}^{s}=V_{1221}^{s}$. Therefore the contributions to $\sigma_{12}(k)$, as well as $\sigma_{21}(k)$, come from three sources: (i) the intersubband $\mathrm{SPE}_{12}$ continuum; the intersubband plasmon modes (ii) $(1,2)$ and (iii) $(1,2)^{\prime}$. In Fig. 4(a) we show $\sigma_{12}(k)$ due only to the $\mathrm{SPE}_{12}$ continuum. The thick and thin lines indicate the $\mathrm{SPE}_{12}$ contributions to $\sigma_{12}(k)$ in the quantum wire of total charge density $N_{e}=N_{1}$ and $N_{2}$, respectively. As discussed before, the $\mathrm{SPE}_{12}$ continuum splits into two parts when the second subband is populated. For $N_{e}=N_{1}$, the onset of scattering $\sigma_{12}(k)$ via emission of an intersubband single-particle excitation in the lower (higher) energy part of the $\mathrm{SPE}_{12}$ continuum occurs at $k \simeq 0.50 \times 10^{6}$ $\mathrm{cm}^{-1}\left(k \simeq 1.46 \times 10^{6} \mathrm{~cm}^{-1}\right)$. The most important contribution of the lower part of the $\mathrm{SPE}_{12}$ is shown in the inset of Fig. 4(a) below the onset of the scattering the higher part. For $\sigma_{21}(k)$ we find no contribution of the single-particle excitations due to restriction of the energy-momentum conservation defined by $\omega_{k}^{21}(q)$. Therefore, electrons cannot transfer from the higher subband to the lower one by emitting a single-particle excitation in the Fermi sea.

In Fig. 4(b), the thick-solid (thick-dashed) line shows the contributions to the inelasticscattering rate $\sigma_{12}(k)\left(\sigma_{21}(k)\right)$ coming from the intersubband plasmon modes $(1,2)$ and $(1,2)^{\prime}$. Again, the thick and thin lines correspond to the results for $N_{e}=N_{1}$ and $N_{2}$, respectively. Notice that the intersubband plasmon modes do not contribute to $\sigma_{21}(k)$ for $k \lesssim k_{c}^{21}$, where $k_{c}^{21} \simeq 1.8 \times 10^{6} \mathrm{~cm}^{-1}$ and $1.31 \times 10^{6} \mathrm{~cm}^{-1}$ for electron density $N_{e}=N_{1}$ and $N_{2}$, respectively. Because the curve $\omega_{k}^{21}(q)$ for $k=k_{c}^{21}$ never intercepts the mode $(1,2)^{\prime}$, the plasmon contribution to the $\sigma_{21}(k)$ comes from the mode $(1,2)$. But both the intersubband plasmon modes contribute to $\sigma_{12}(k)$. The onset of scattering $\sigma_{12}(k)$ due to the emission of 
the plasmon mode $(1,2)$ occurs at the threshold $k_{c}^{12} \simeq 3.1 \times 10^{6} \mathrm{~cm}^{-1}\left(2.61 \times 10^{6} \mathrm{~cm}^{-1}\right)$ for $N_{e}=N_{1}\left(N_{2}\right)$. Indeed, we verified that the curve $\omega_{k}^{12}(q)$ intercepts the plasmon mode $(1,2)$ for $k \geq k_{c}^{12}$ and the mode $(1,2)^{\prime}$ for all values of $k$ except $k=k_{F 1}$. As a matter of fact, we see the scattering rate $\sigma_{12}(k)$ vanishing at $k=k_{F 1}$ due to restrictions of the momentum-energy conservation dictated by the step functions in Eq. (四). In the inset, we show the most relevant contribution to $\sigma_{12}(k)$ coming from the plasmon mode $(1,2)^{\prime}$.

We summarize the above results by plotting the total inelastic-scattering rate

$$
\sigma_{n}(k)=\sum_{n^{\prime}=1,2} \sigma_{n n^{\prime}}(k)
$$

in Figs. 5 and 6, respectively, for an electron in the subband $n=1$ and 2 in the quantum wire of total charge density $N_{e}=N_{1}$. The scale in the right-hand side of Fig. 5 (Fig. 6) is enlarged 15 (5) times as compared to that in the left. The symbols indicate the contributions from the different scattering mechanisms. The circles stand for the contribution coming from the emission of a plasmon mode $(2,2)$, while the squares represent the contribution coming from the emission of the plasmon mode $(1,1)$. The filled (open) triangles-left stand for the contribution coming from the emission of a intersubband plasmon mode $(1,2)\left((1,2)^{\prime}\right)$. The open (filled) diamonds represent the contribution coming from single-particle excitations inside the $\mathrm{SPE}_{22}\left(\mathrm{SPE}_{11}\right)$ continuum. Finally, open (filled) triangles-up in Fig. 5 represent the contribution coming from the single-particle excitations in the lower (higher) part of the $\mathrm{SPE}_{12}$ continuum. These symbols show the complexity of various intra- and inter-subband single particle and collective mode contributions to the scattering rate of an electron which might be scattered either to unoccupied states in its original subband or to those in a different subband. In contrast to $\sigma_{2}(k)$, we see $\sigma_{1}(k)$ being finite at $k=0$ due to the possibility of emission of a single-particle excitation within the $\mathrm{SPE}_{22}$ continuum. As $k$ increases, the plasmon mode $(1,2)^{\prime}$ starts to contribute to $\sigma_{1}(k)$ and then, as we discussed above, all excitations in the phase space contribute to scattering. In Fig. 5, the open triangles-up indicate the contribution coming from the single-particle excitations inside the lower energy part of the $\mathrm{SPE}_{12}$ continuum. Notice that we neglected such a contribution in the right-hand 
side since it is irrelevant at that scale. When the second subband is empty for $N_{e}=N_{2}$, we should see neither the contributions coming from the plasmon modes $(1,2)^{\prime}$ and $(2,2)$ nor those from the $\mathrm{SPE}_{22}$ continuum.

\section{Hot electron lifetimes}

We now discuss the hot electron lifetime

$$
\tau_{E, n}=\frac{2}{\sigma_{n}(k)}
$$

of an energetic hot electron injected in a subband $n$ with a kinetic energy $E=\hbar^{2} k^{2} / 2 m^{*}$ above the Fermi energy $E_{F n}=\hbar^{2} k_{n}^{2} / 2 m^{*}$ in the subband. It is well-known that this lifetime $(\hbar=1)$ can be written as Eq. (13) since $\sigma_{n}(k)$ is the absolute value of the imaginary part of the self-energy of an electron in the subband $n$. 19 In Figs. 7(a) and 7(b) we show the lifetimes $\tau_{E, 1}$ and $\tau_{E, 2}$ of hot electrons injected in the first and the second subband, respectively, as a function of the total electron density $N_{e}$ in the quantum wire. The vertical thin line at $N_{e}=0.63 \times 10^{6} \mathrm{~cm}^{-1}$ indicates the onset of the population of the second subband for our quantum wire parameters. The thick-solid (thick-dashed) lines indicates the lifetime of an injected "hot electron" in the conduction band with total energy $E_{T 1}=E_{2}+\omega_{0}$ $\left(E_{T 2}=E_{2}+3 \omega_{0}\right)$, where $E_{2}$ is the bottom of the second subband. The symbols stand for the same meaning as in Figs. 5 and 6, and show the contributions of the different chargedensity excitations to the total lifetime. We see the plasmon mode $(1,1)$ making the most important contribution (solid squares) to $\tau_{E, 1}$ for $E=E_{T 1}$ (thick solid lines) at low densities. The decreasing of $\tau_{E_{T 1}, 1}$ as $N_{e}$ increases indicates the hot-electron relaxation via emission of a plasmon mode $(1,1)$. The single-particle excitations inside the $\mathrm{SPE}_{12}$ continuum (filled triangles-up) start to contribute to $\tau_{E_{T 1}, 1}$ as the onset of the scattering via emission of the plasmon mode $(1,1)$ vanishes at $N_{e} \gtrsim 0.35 \times 10^{6} \mathrm{~cm}^{-1}$. For $E=E_{T 2}$, however, the $\mathrm{SPE}_{12}$ is the main contribution to $\tau_{E, 1}$ at very low densities. With increasing density, scattering due to the plasmon mode $(1,1)$ becomes dominant until $N_{e} \simeq 0.83 \times 10^{6} \mathrm{~cm}^{-1}$. The occupation 
of the second subband only leads to a small contribution by the intersubband plasmon $(1,2)^{\prime}$ (triangles-left) to $\tau_{E, 1}$. Although the onset of the scattering by the plasmon mode $(1,2)$ is achieved at low density $N_{e}$, its contribution to $\tau_{E, 1}$ is so small that we cannot observe it in the figure. The contribution coming from the $\mathrm{SPE}_{22}$ is also irrelevant in this situation. As discussed before, this excitation exists as the second subband is occupied but its contribution to scattering vanishes for momentum $k \geqslant k_{F 2}$. Here, we are dealing with a hot-electron of energy $E \gg\left(\hbar k_{F 2}\right)^{2} / 2 m^{*}$ for which relaxation via the emission of single-particle excitations inside the $\mathrm{SPE}_{22}$ continuum is not allowed.

In Fig. 7(b), we see that the emission of the plasmon mode $(1,2)$ (filled triangles-left), at very low densities, is the main contribution to the total lifetime $\tau_{E, 2}$ for both values of $E$. As $N_{e}$ increases, the hot-electron scattering via emission of the plasmon mode $(1,1)$, as well as the emission of single-particle excitations inside $\mathrm{SPE}_{11}$ continuum (filled diamonds), start to contribute to $\tau_{E, 2}$. For densities greater than $N_{e} \simeq 0.36 \times 10^{6} \mathrm{~cm}^{-1}\left(N_{e} \simeq 0.44 \times 10^{6} \mathrm{~cm}^{-1}\right)$ the onset of scattering via the emission of the plasmon mode $(1,1)((1,2))$ vanishes, so that only single-particle excitations inside the $\mathrm{SPE}_{11}$ continuum (filled diamonds) are responsible for the hot-electron relaxation in the second subband. For $E=E_{T 2}$, the onset of scattering via the emission of the plasmon mode $(1,2)$ occurs at densities $N_{e}>10^{6} \mathrm{~cm}^{-1}$. As a result, this mode contributes to $\tau_{E, 2}$ for all values of $N_{e}$ shown in the figure.

At this point, we should briefly comment on the role of the phenomenological damping constant $\gamma$ on our numerical results since we have used $\gamma=10^{-3} \mathrm{meV}$ throughout this paper. In contrast to Figs. 5 and 6 , where effects of $\gamma\left(=10^{-3}\right)$ are vanishingly small, a finite $\gamma$ has some effect in Fig. 7. Notice that, for extremely clean systems $(\gamma=0)$, the contributions to both $\tau_{E, n}$, coming from the plasmon mode $(1,1)$ (square lines), should go to infinity threshold due to the singular nature of 1D density of states. Impurity scattering through a finite $\gamma$ suppresses this divergence by smoothing the 1D density of states. Similar behavior should occur for the contribution to $\tau_{E, 2}$ coming from the emission of the mode $(1,2)$ (filled triangles-left) in Fig. 7(b). In fact, effects due to finite values of $\gamma$ on both hotelectron lifetimes can be identified in the lines indicating the contribution of the plasmon 
modes $(1,1)$ and $(1,2)$. They do not go to infinity since we are using $\gamma=10^{-3} \mathrm{meV}$, which is enough to suppress the $1 \mathrm{D}$ density of states singularity on the scales of these figures. We see, however, that these effects are irrelevant for the total hot-electron lifetime when the emission of single-particle excitations are taken into account. For the value of $\gamma$ assumed in this paper, the relaxation of hot electrons is mainly due to emission of charge-density excitations in the Q1D Fermi sea.

\section{SUMMARY}

Within the GW approximation, we have calculated the inelastic Coulomb scattering rates and lifetimes of an injected electron in a symmetric confinement two-subband quantum wire at zero temperature. These rates are directly related to the dynamically screened Coulomb potential which has been calculated within the RPA. We chose a quantum wire with symmetric confinement potential in which the intra- and intersubband excitations do not interact with each other. We obtain the effects of the population of second subband on the inelastic Coulomb scattering rate. We separately identified the contributions to the intrasubband and intersubband inelastic-scattering rates due to different intrasubband and intersubband excitations in the individual subbands of the Q1D electron system. We find the emission of an intrasubband plasmon in the first subband to be the most important contribution to the inelastic-scattering rate, although the single-particle excitations as well as plasmon modes in the second subband also contribute to the intrasubband and intersubband scattering of an electron in the two-subband quantum wire. We found that the inelastic scattering from the first to the second subband occurs through the emission of either intersubband plasmon modes or intersubband single-particle excitations, whereas the scattering from second to first subband only occurs via the emission of the higher energy intersubband plasmon mode. We also calculate the lifetime of hot electrons as a function of the total charge density in the two-subband quantum wire, identifying the contributions of plasmons and single particle excitations in each subband to the hot electron lifetime. 


\section{ACKNOWLEDGMENTS}

We would like to thank E.H. Hwang for very useful discussions. The work of MRST is supported by FAPESP, Brazil. The work at Maryland is supported by the US-ARO and US-ONR. G-QH acknowledgs $C N P q$ from Brazil for partial support. 


\section{REFERENCES}

[1] A. R. Goñi, A. Pinczuk, J. S. Weiner, J.M. Calleja, B. S. Dennis, L. N. Pfeiffer, and K. W. West, Phys. Rev. Lett. 67, 3298 (1991).

[2] S. Das Sarma and W.Y.Lai, Phys. Rev. B 32, 1401 (1985); Q.P. Li and S. Das Sarma, Phys. Rev. B 43, 11768 (1991).

[3] J.M.Calleja, B. S. Dennis, A. Pinczuk, S. Schittrink, L. N. Pfeiffer, K. W. West, J.F. Muller, and A.E. Ruckestein, Surf. Sci. 263, 346 (1992).

[4] W. Wegscheider, L.N. Pfeiffer, M.M. Dignam, A. Pinczuk, K.W. West, S.L. Mccall, and R. Hull, Phys. Rev. Lett. 71, 4071 (1999).

[5] S. Das Sarma and D.W. Wang, Phys. Rev. Lett. 83, 816 (1999).

[6] S.Q. Murphy, J.P. Eisenstein, L.N. Pfeiffer, and K.W. West, Phys. Rev. B 52, 14825 (1995).

[7] S. Xu, J. Cao, C. C. Miller, D. A. Mantell, R. J. D. Miller, and Y. Gao, Phys. Rev. Lett. 76483 (1996).

[8] Lian Zheng and S. Das Sarma, Phys. Rev. B 53, 9964 (1996).

[9] R. Jalabert and S. Das Sarma, Phys. Rev. B 40, 9723 (1989); Paul Sotirelis, Paul von Allmen and Karl Hess, Phys. Rev. B 47, 12744 (1993).

[10] W. I. Friesen and B. Bergersen, J. Phys. C 13, 6627 (1980).

[11] A. Gold and A. Ghazali, Phys. Rev. B 41, 7626 (1990).

[12] W.Y. Lai, A. Kobayashi and S. Das Sarma, Phys. Rev. 34, 7380 (1986).

[13] Lian Zheng and S. Das Sarma, Phys. Rev. B 54, 13908 (1996).

[14] S. Das Sarma and E.H. Hwang, Phys. Rev. B 59, 10730 (1999).

[15] B. Vinter, Phys. Rev. B 15, 3947 (1977). 
[16] E.H. Hwang, B. Yu-Kuang and S. Das Sarma, Phys. Rev. B 54, 4996 (1996).

[17] B. Yu-Kuang Hu and S. Das Sarma, Phys. Rev. B 48, 5469 (1993).

[18] Marcos R.S. Tavares and Guo-Qiang Hai, Phys. Rev. 61, 7564 (2000).

[19] For review, see G. D. Mahan, Many-Particle Physics Plenum, New York, 1981. 2nd edition.

[20] N. D. Mermin, Phys. Rev. B 1, 2362 (1970). 


\section{FIGURES}

FIG. 1. Dispersion relation of both (a) inter- and (b) intrasubband charge-density excitations in a quantum wire of $\mathrm{N}_{e}=\mathrm{N}_{1}=10^{6} \mathrm{~cm}^{-1}$ with $\omega_{0}=5.37 \mathrm{meV}$. The shadow areas indicate the (a) inter- and (b) intrasubband single-particle continua. Both subbands are occupied.

FIG. 2. Dispersion relation of both inter- and intrasubband charge-density excitations in the same quantum wire of $\mathrm{N}_{e}=\mathrm{N}_{2}=0.4 \times 10^{6} \mathrm{~cm}^{-1}$. The shadow areas indicate the (a) intersubband and (b) intrasubband single-particle continua. Only the lowest subband is occupied

FIG. 3. Intrasubband inelastic-scattering rates $\sigma_{11}$ (solid lines) and $\sigma_{22}$ (dashed lines) due to emission of intrasubband (a) single-particle and (b) collective excitations. Thick (thin) lines represent results for our quantum wire of density $\mathrm{N}_{e}=\mathrm{N}_{1}\left(\mathrm{~N}_{e}=\mathrm{N}_{2}\right)$.

FIG. 4. Intersubband inelastic-scattering rates $\sigma_{12}$ (solid lines) and $\sigma_{21}$ (dashed lines) due to intersubband (a) single-particle and (b) collective exciatations. Thick (thin) lines represent results for a quantum wire of density $\mathrm{N}_{e}=\mathrm{N}_{1}\left(\mathrm{~N}_{e}=\mathrm{N}_{2}\right)$. Inset in part (a) shows the $\sigma_{12}$ due to only those single-particle excitations in the lower energy part of the $\mathrm{SPE}_{12}$ continuum. Inset in part (b) shows $\sigma_{12}$ only due to the plasmon mode $(1,2)^{\prime}$.

FIG. 5. Inelastic-scattering rate $\sigma_{n}(k)$ of electrons in the first subband $(\mathrm{n}=1)$. The density in the quantum wire is $\mathrm{N}_{e}=\mathrm{N}_{1}$.

FIG. 6. Inelastic-scattering rate $\sigma_{n}(k)$ of electrons in the second subband $(\mathrm{n}=2)$. The density in the quantum wire is $\mathrm{N}_{e}=\mathrm{N}_{1}$.

FIG. 7. Hot electron lifetimes (a) $\tau_{E, 1}$ and (b) $\tau_{E, 2}$ as a function of the total density $N_{e}$ in the quantum wire. Thick solid (dashed) lines are the total lifetime of a hot electron with $E=E_{T 1}$ $\left(E=E_{T 2}\right)$. The thin lines indicate all sort of contribution to the total lifetime. The symbols stand for the same contributions as in Figs. 5 and 6. 


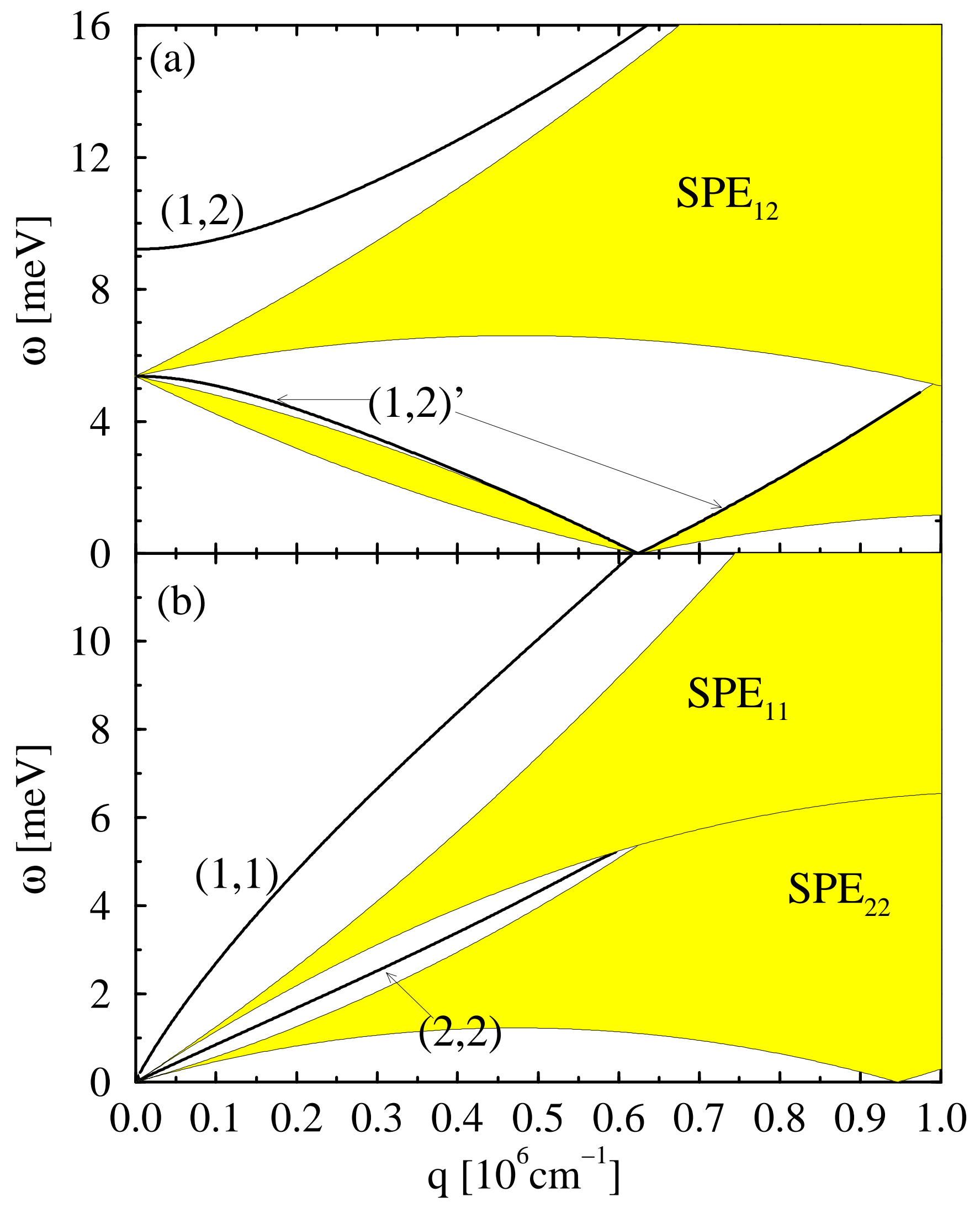




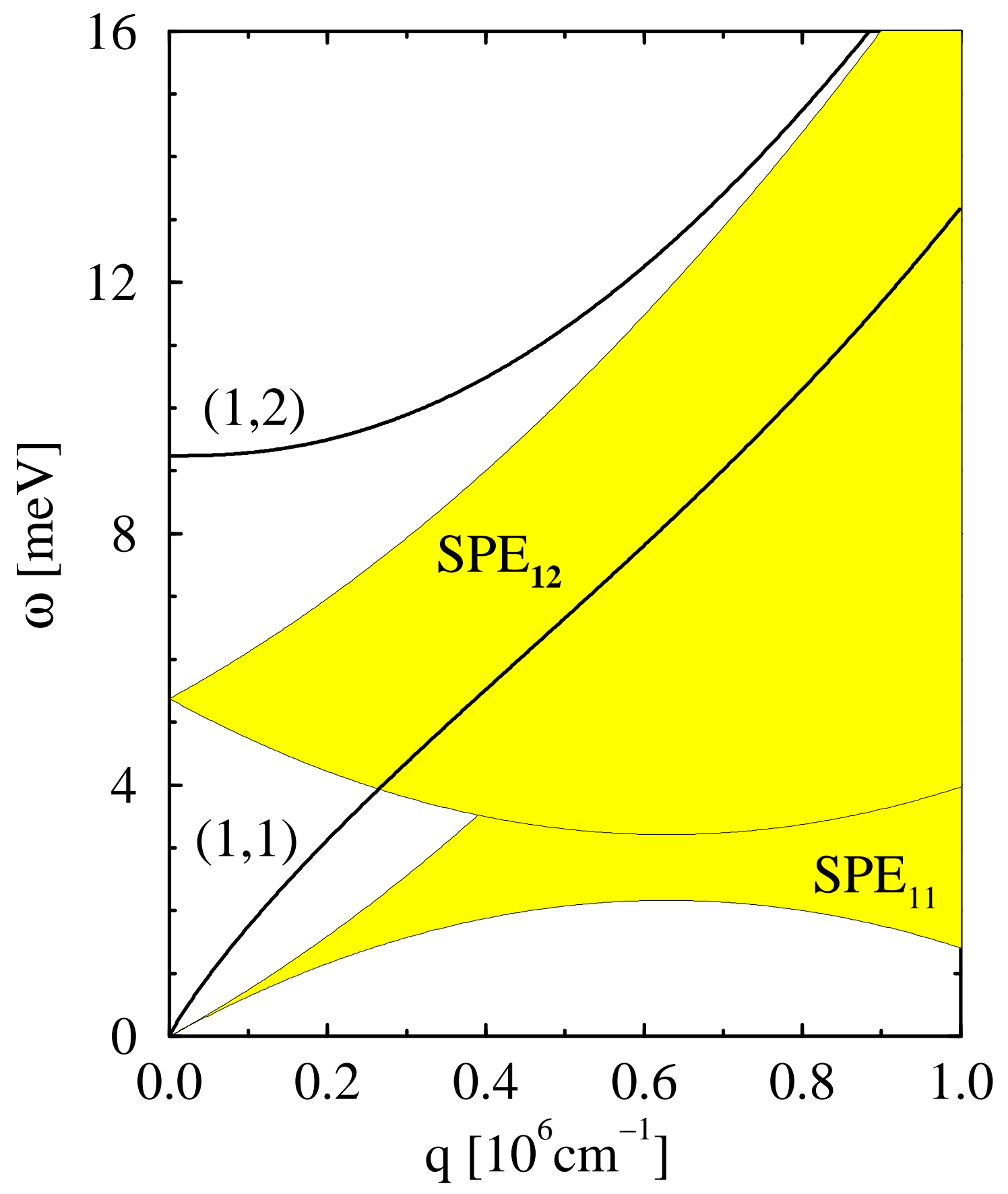




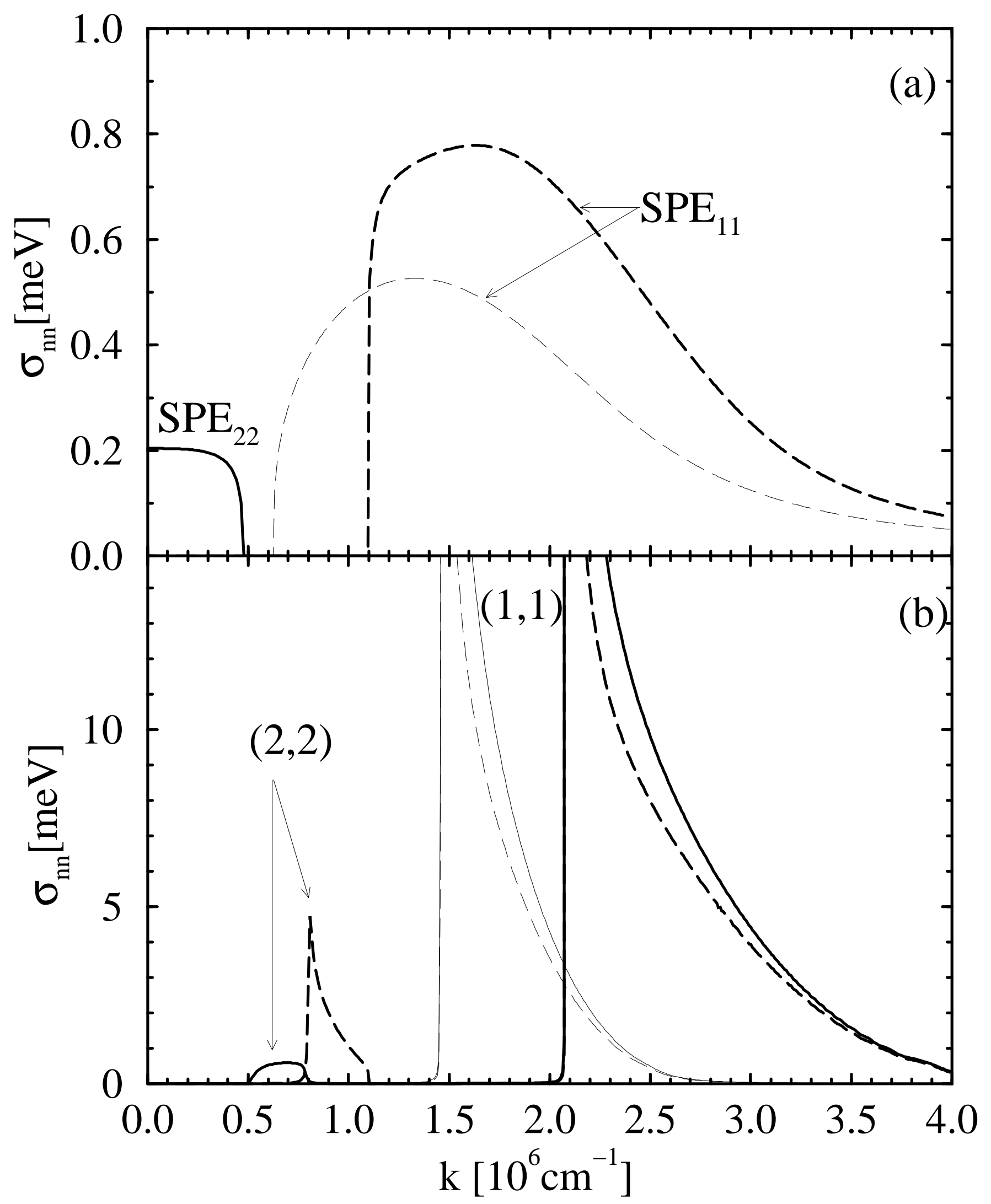




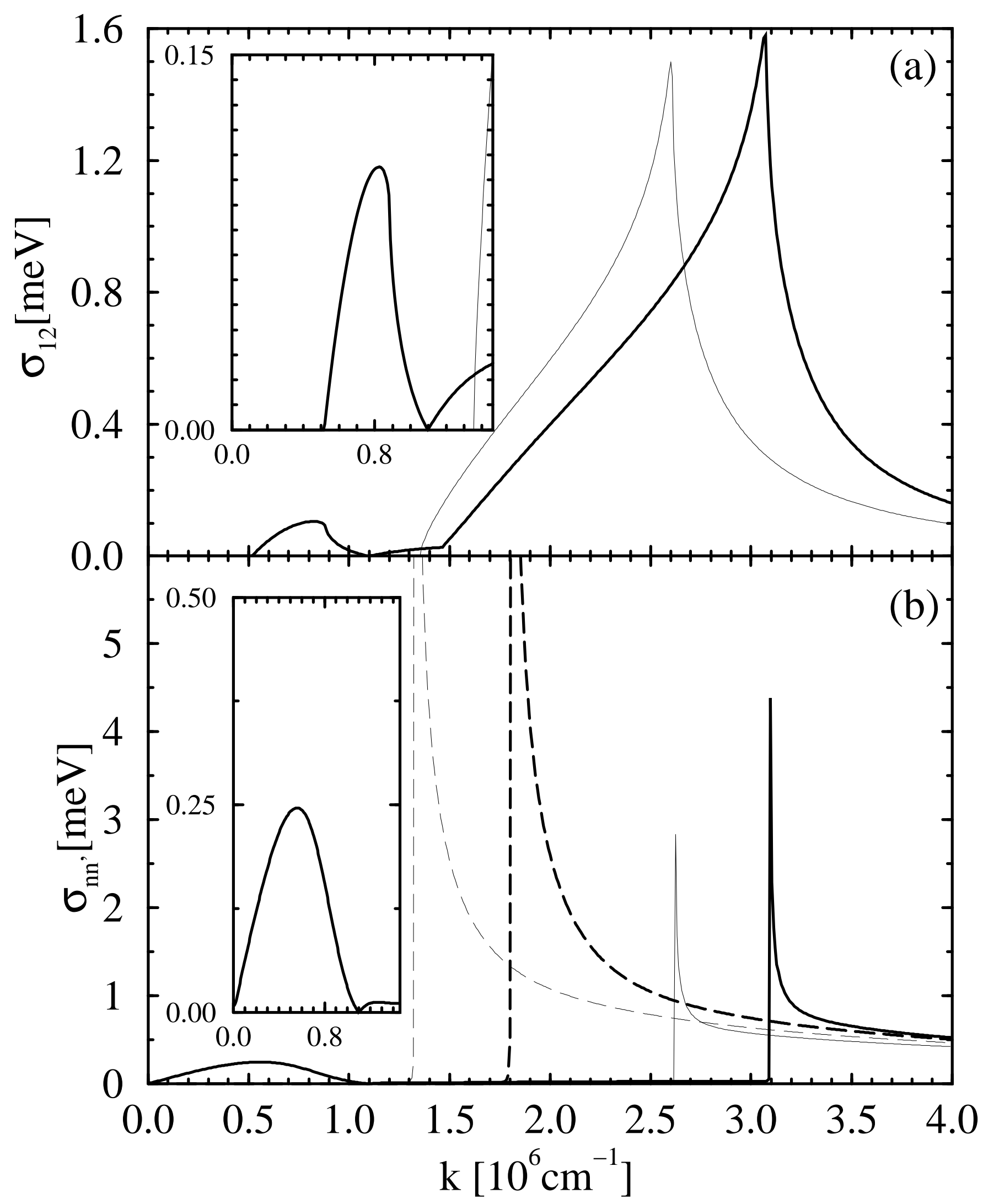




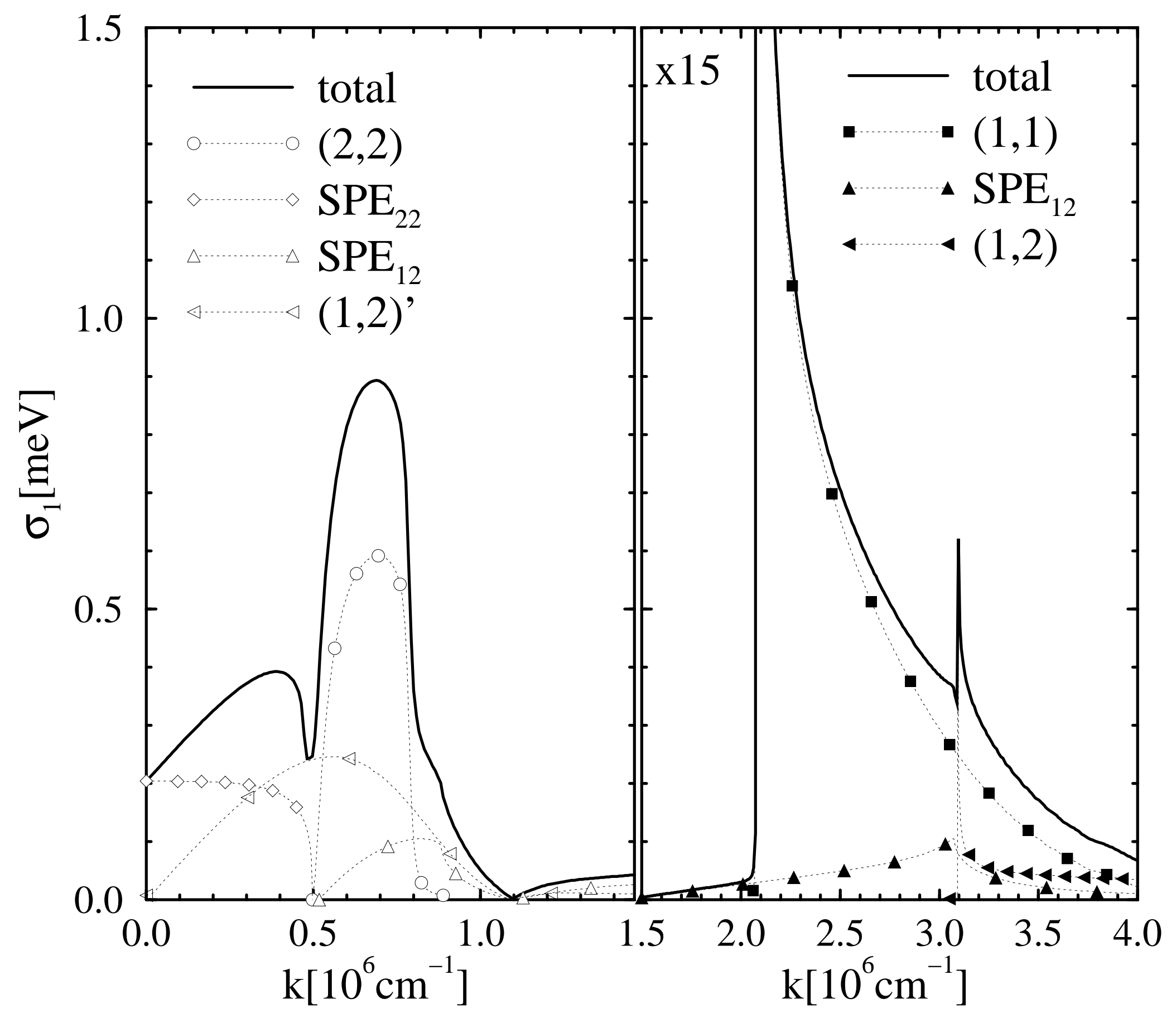




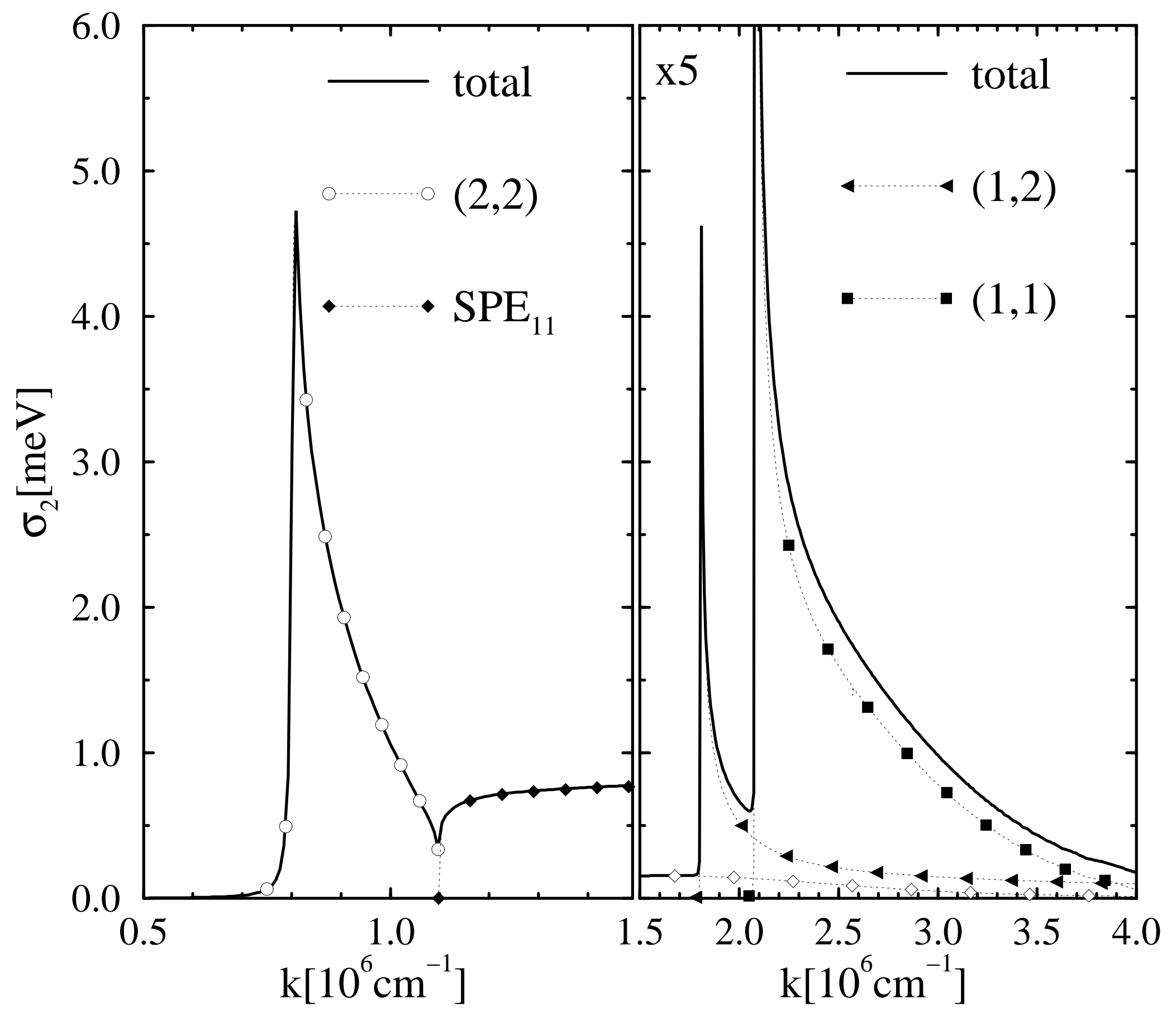




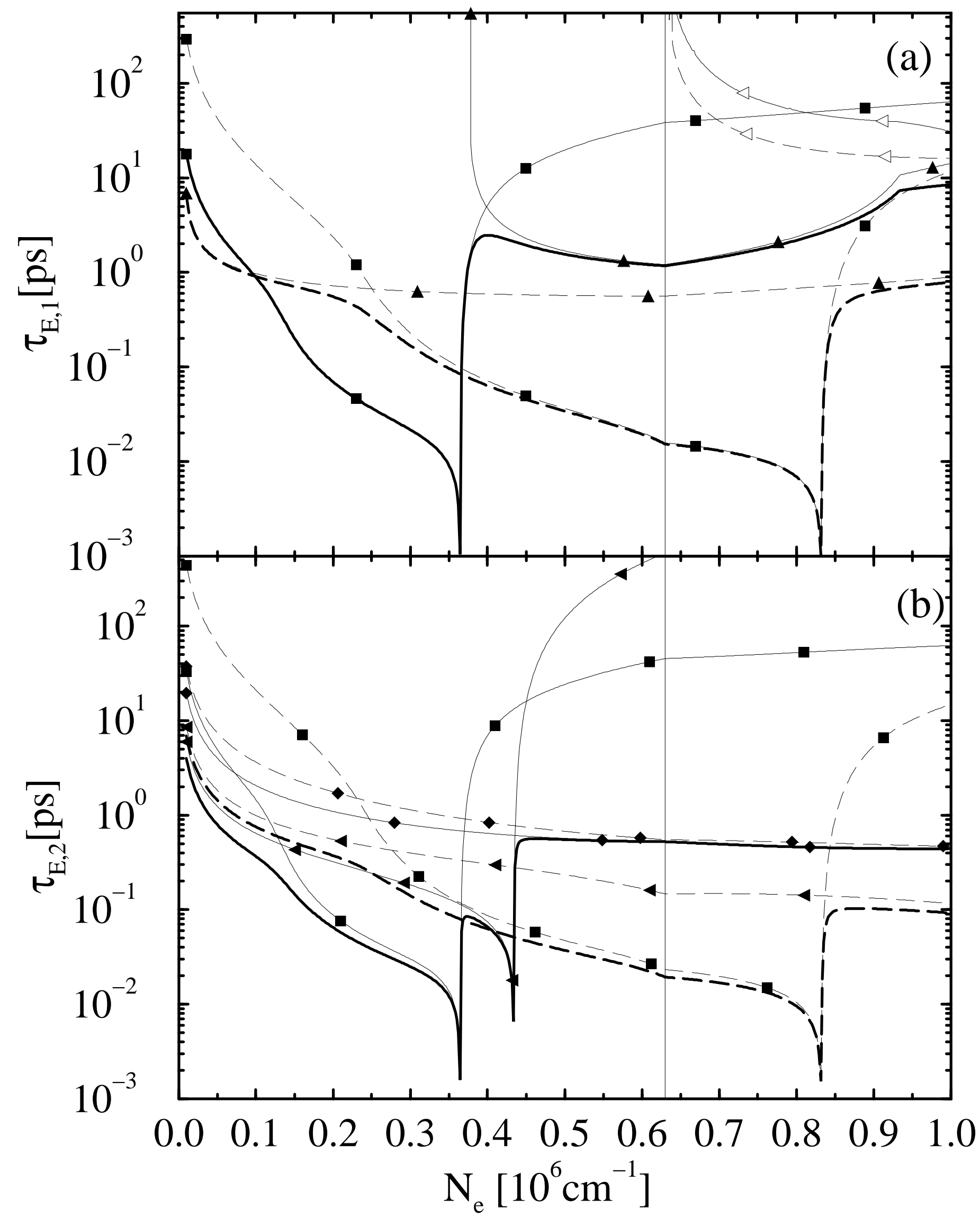

